\title{
Peripheral CD4+ naïve/memory ratio is an independent predictor of survival in non-small cell lung cancer
}

\author{
Peng Yang ${ }^{1}$, Junhong $\mathrm{Ma}^{1}$, Xin Yang ${ }^{2}$ and Wei Li ${ }^{1}$ \\ ${ }^{1}$ Department of Thoracic Surgery, Linyi People's Hospital, Linyi 276000, China \\ ${ }^{2}$ The Statistics Research and Consulting Laboratory, Culverhouse College of Commerce and Business Administration, The \\ University of Alabama, Tuscaloosa, AL 35487, USA \\ Correspondence to: Wei Li, email: willi1973@163.com \\ Keywords: non-small cell lung cancer, T cells, CD45RA, CD45RO, survival \\ Received: May 17, $2017 \quad$ Accepted: June 19, $2017 \quad$ Published: July 18, 2017 \\ Copyright: Yang et al. This is an open-access article distributed under the terms of the Creative Commons Attribution License \\ 3.0 (CC BY 3.0), which permits unrestricted use, distribution, and reproduction in any medium, provided the original author and \\ source are credited.
}

\section{ABSTRACT}

Background: To investigate the clinical significance of naïve $\mathrm{T}$ cells, memory T cells, CD45RA+CD45RO+ $T$ cells, and naïve/memory ratio in non-small cell lung cancer (NSCLC) patients.

Methods: Pretreatment peripheral blood samples from 76 NSCLC patients and 28 age- and sex-matched healthy volunteers were collected and tested for immune cells by flow cytometry. We compared the expression of these immune cells between patients and healthy controls and evaluated their predictive roles for survival in NSCLC by cox proportional hazards model.

Results: Decreased naïve CD4+ T cells, naïve CD8+ T cells, CD4+ naïve/memory ratios and CD4+CD45RA+CD45RO+ $T$ cells, and increased memory CD4+ $T$ cells, were observed in 76 NSCLC patients compared to healthy volunteers. Univariate analysis revealed that elevated CD4+ naïve/memory ratio correlated with prolonged progression-free survival $(P=0.013)$. Multivariate analysis confirmed its predictive role with a hazard ratio of 0.35 (95\% confidence interval, $0.19-0.75, P=0.012)$.

Conclusions:Peripheral CD4+ naïve/memory ratio can be used as a predictive biomarker in NSCLC patients and used to optimize personalized treatment strategies.

\section{INTRODUCTION}

Lung cancer is the most common malignant cancer and the leading cause of cancer death worldwide [1]. Surgery with or without postoperative radiochemotherapy is the standard therapy for operable non-small cell lung cancer (NSCLC) patients with stage I-III A [2,3]. For non-operable NSCLC patients, chemotherapy, targeted therapy, radiotherapy and their combination are utilized $[4,5]$. Natural innate and adaptive immunity play important roles in cancer development and interact with cancer therapeutics $[6,7]$. Based on this mechanism, we hypothesized that the pretreatment systemic immune status could influence the effects of cancer therapeutics and consequently affect patients' clinical outcomes. Many studies have investigated the prognostic value of $\mathrm{CD} 4+\mathrm{T}$ cells, $\mathrm{CD} 8+\mathrm{T}$ cells, regulatory $\mathrm{T}$ cells and $\mathrm{B}$ cells [8-12]. Nevertheless, very little is known of the predictive roles of peripheral naïve $\mathrm{T}$ cells, memory $\mathrm{T}$ cells and CD45RA+CD45RO $+\mathrm{T}$ cells in NSCLC patients.

CD45RA $+\mathrm{T}$ cells are identified as naïve $\mathrm{T}$ lymphocytes that have not responded to antigen; they will be activated by the stimulation of antigen and differentiate to effector cells against tumor cells. CD45RO is the marker of memory $\mathrm{T}$ lymphocytes which have responded to tumor antigen and will solicit immune response by the re-exposure of antigen [13-15]. T cells coexpressing CD45RA and CD45RO are special cells in the process of transformation from naïve cells to memory cells [16]. Hara et al. [17] found no differences in the proportions of naïve CD4+ $\mathrm{T}$ cells, memory CD4+ $\mathrm{T}$ cells, and the CD4+ naïve/memory ratios between NSCLC patients and healthy controls. Similarly, there were no significant 
differences of naïve CD8+ cells and memory CD8+ cells [18]. Two very recent studies proved that high levels of CD45RO (+) tumor-infiltrating lymphocytes were associated with better survival in lung cancer patients $[19,20]$.

Thus, the purpose of this study was to investigate the clinical significance of peripheral naïve $\mathrm{T}$ cells, memory $\mathrm{T}$ cells, CD45RA $+\mathrm{CD} 45 \mathrm{RO}+\mathrm{T}$ cells and naïve/memory ratio in NSCLC patients.

\section{RESULTS}

\section{Clinicopathologic characteristics}

Clinicopathologic characteristics of 76 NSCLC patients are shown in Table 1. Among them, 41 received radical surgery and 35 were treated with radiotherapy combined with chemotherapy/targeted therapy. Tumors with epidermal growth factor receptor (EGFR) mutation occurred in 4 patients; anaplastic lymphoma kinase (ALK) gene fusion occurred in 2 patients. nineteen patients had evidence of distant metastases at diagnosis; there were 5 with lung metastases, 3 with brain metastases, 6 with bone metastases, and 5 with adrenal metastasis.

\section{The expression of immune cells in 76 NSCLC patients}

Lung cancer patients expressed significantly decreased proportions of naïve CD4+ T cells, naïve CD8+ $\mathrm{T}$ cells, CD4+ naïve/memory ratios, and $\mathrm{CD} 4+\mathrm{CD} 45 \mathrm{RA}+\mathrm{CD} 45 \mathrm{RO}+\mathrm{T}$ cells and increased memory CD4+ $\mathrm{T}$ cells compared to healthy controls (Figure 1, Table 2). In addition, a similar trend occurred in $\mathrm{CD} 8+$ naïve/memory ratios, $\mathrm{CD} 8+\mathrm{CD} 45 \mathrm{RA}+\mathrm{CD} 45 \mathrm{RO}+\mathrm{T}$ cells and memory CD8+ $\mathrm{T}$ cells, but the differences did not reach statistical significance.

\section{Correlation of immune cells with clinicopathologic characteristics}

Table 3 summarizes the relationships between immune cells and clinicopathological characteristics in the 76 NSCLC patients. In never-smokers, high proportions of naïve CD4+ $\mathrm{T}$ cells and low memory CD4+ T cells were observed. Similarly, there was a trend in younger patients for these findings although they did not achieve statistical significance. In patients with good performance status, decreased naïve CD8 $+\mathrm{T}$ cells and $\mathrm{CD} 8+$ naïve/memory and increased memory CD8+ $\mathrm{T}$ cells were found.

\section{Increased CD4+ naïve/memory ratio associated with better progression-free survival}

Univariate analysis of immune parameters and clinicopathologic parameters are shown in Table 4. Interestingly, patients with a high level of CD4+ naïve/ memory ratio had a better PFS $(\mathrm{HR}=0.47,95 \% \mathrm{CI}=$ $0.20-0.92, P=0.013$, Figure 2A). In contrast, elevated CD4+CD45RA + CD45RO $+\mathrm{T}$ cells predicted poor PFS with a robust trend toward significance $(\mathrm{HR}=2.71$, 95\%CI $=1.15-6.36, P=0.058$, Figure 2B). Besides, patients with an increase of memory CD4+ T cells may have a poor PFS $(\mathrm{HR}=1.82,95 \% \mathrm{CI}=0.78-4.25, P=$ 0.138 , Table 4).

In addition to immune parameters, we found other parameters associated with clinical outcomes. Tumor differentiation, nodal stage, clinical stage and the Eastern Cooperative Oncology Group performance status correlated with the patients' clinical outcome (Table 4).

\section{CD4+ naïve/memory ratio independently correlated with progression-free survival}

Multivariate cox proportional hazard models revealed that increased CD4+ naïve/memory ratio was independently associated with better PFS (HR $=0.35$, $95 \% \mathrm{CI}=0.19-0.75, P=0.012$, Table 5). In addition, nodal stage and clinical stage were independently correlated with the progression-free survival of 76 NSCLC patients.

\section{DISCUSSION}

To the best of our knowledge, this is a study with the largest sample size to investigate the predictive roles of peripheral naïve/memory ratio, naïve cells, memory cells, and CD45RA+CD45RO $+\mathrm{T}$ cells in patients with NSCLC. The most important findings were that elevated CD4+ naïve/memory ratio was independently correlated with prolonged PFS. Moreover, lower percentages of naïve CD4+ $\mathrm{T}$ cells, naïve CD8+ T cells, CD4+ naïve/ memory ratios, and CD4+CD45RA $+\mathrm{CD} 45 \mathrm{RO}+\mathrm{T}$ cells, and increased memory CD4+ T cells, occurred in NSCLC patients compared to healthy controls.

Hara et al. [17] investigated the correlation between CD4+ naïve/memory ratio and survival after surgery in NSCLC patients and proved this possible relationship; however, there were only 8 patients in each group. In the present study, we found that elevated CD4+ naïve/memory ratio was independently associated with prolonged PFS in NSCLC patients; elevated peripheral memory $\mathrm{CD} 4+$ cells may indicate poor survival, which is contrary to the role of CD45RO+ 
Table 1: Clinicopathologic characteristics of 76 NSCLC patients

\begin{tabular}{|c|c|}
\hline Parameter & $\mathrm{N}=76(\%)$ \\
\hline Age(years) & $64.5(35-87)$ \\
\hline \multicolumn{2}{|l|}{ Gender } \\
\hline Male & $54(71.1)$ \\
\hline Female & $22(28.9)$ \\
\hline \multicolumn{2}{|l|}{ ECOG } \\
\hline 0 & $32(42.1)$ \\
\hline $1-2$ & $44(57.9)$ \\
\hline \multicolumn{2}{|l|}{ Smoking history } \\
\hline Yes & $50(65.8)$ \\
\hline No & $26(34.2)$ \\
\hline \multicolumn{2}{|l|}{ Histology } \\
\hline Squamous cell carcinoma & $37(48.7)$ \\
\hline Adenocarcinoma & $39(51.3)$ \\
\hline \multicolumn{2}{|l|}{ Differentiation } \\
\hline Well/moderate & $48(63.2)$ \\
\hline Poor & $23(30.3)$ \\
\hline Unknown & $5(6.5)$ \\
\hline \multicolumn{2}{|l|}{ T stage } \\
\hline 1 & $20(26.3)$ \\
\hline 2 & $32(42.1)$ \\
\hline 3 & $11(14.5)$ \\
\hline 4 & $13(17.1)$ \\
\hline \multicolumn{2}{|l|}{$\mathrm{N}$ stage } \\
\hline 0 & $21(27.6)$ \\
\hline 1 & $24(31.6)$ \\
\hline 2 & $19(25.0)$ \\
\hline 3 & $12(15.8)$ \\
\hline \multicolumn{2}{|l|}{ Clinical stage (TNM) } \\
\hline I & $8(10.5)$ \\
\hline II & $16(21.1)$ \\
\hline III & $33(43.4)$ \\
\hline IV & $19(25.0)$ \\
\hline
\end{tabular}

NSCLC=non-small cell lung cancer, ECOG=Eastern Cooperative Oncology Group. 

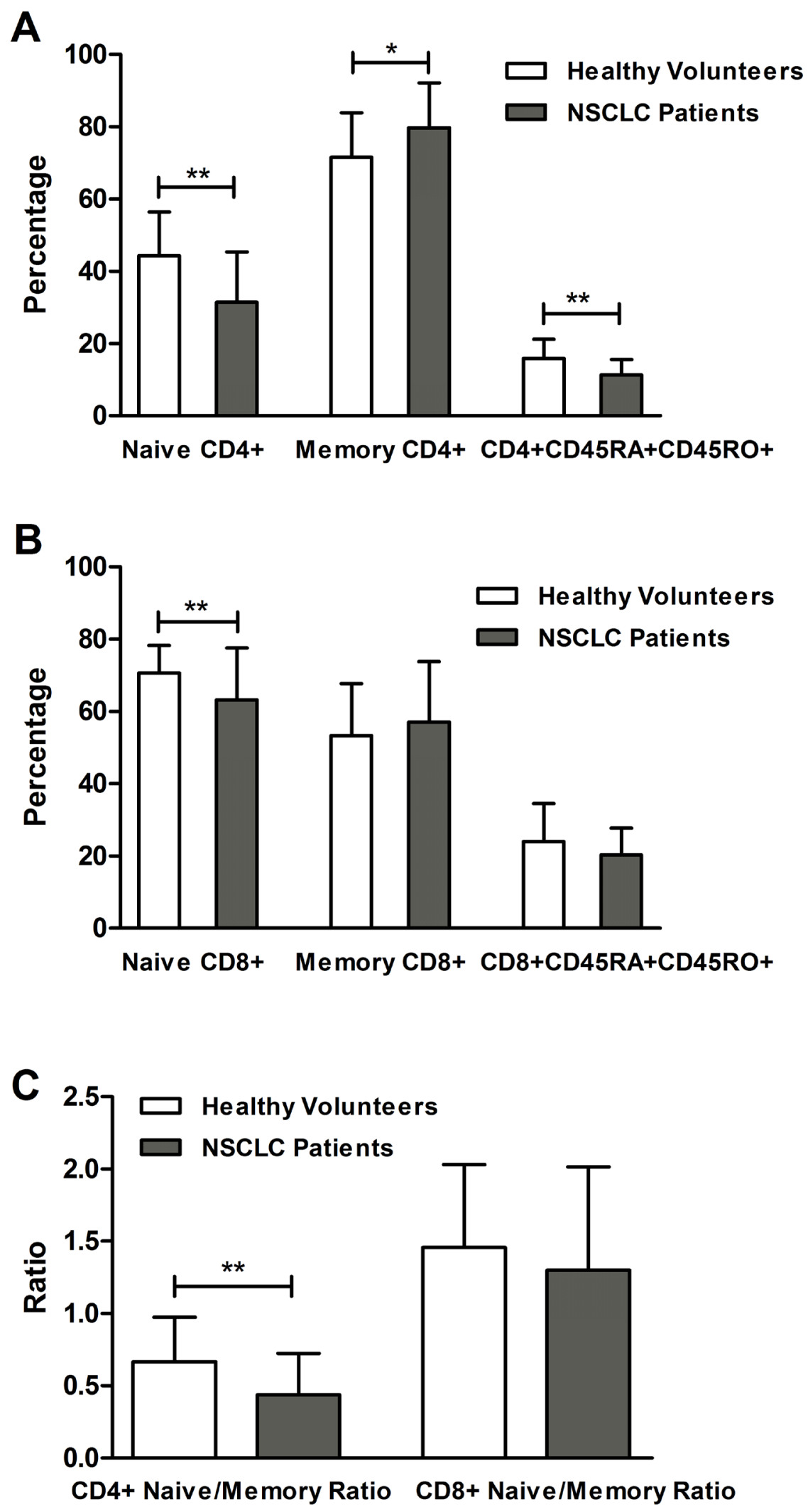

Figure 1: Differences in the proportions of CD45RA and CD45RO marked $T$ cells between NSCLC patients and healthy controls. In NSCLC, there are (A) decreased naïve CD4+ T cells and CD4+CD45RA+CD45RO+ T cells, and increased memory CD4+ T cells; (B) reduced naïve CD8+ T cells; (C) low CD4+ naïve/memory ratio. ${ }^{*} P<0.05 ;{ }^{* *} P<0.01$. 
Table 2: Differences of immune cells between NSCLC patients and healthy volunteers

\begin{tabular}{|c|c|c|c|}
\hline \multirow{2}{*}{ Variable } & Healthy volunteers & NSCLC & \multirow{2}{*}{$P$-value } \\
\hline & $\operatorname{Mean} \pm$ SD & Mean \pm SD & \\
\hline Naïve CD4+ & $44.26 \pm 12.21$ & $31.46 \pm 15.26$ & 0.002 \\
\hline Memory CD4+ & $71.52 \pm 12.35$ & $79.83 \pm 13.71$ & 0.025 \\
\hline $\mathrm{CD} 4+\mathrm{CD} 45 \mathrm{RA}+\mathrm{CD} 45 \mathrm{RO}+$ & $15.85 \pm 5.32$ & $11.38 \pm 4.49$ & 0.001 \\
\hline $\mathrm{CD} 4+$ naïve/memory ratio & $0.67 \pm 0.31$ & $0.44 \pm 0.34$ & 0.009 \\
\hline Naïve CD8+ & $70.66 \pm 7.62$ & $61.15 \pm 15.02$ & 0.045 \\
\hline Memory CD8+ & $53.35 \pm 14.35$ & $57.03 \pm 16.3$ & 0.451 \\
\hline $\mathrm{CD} 8+\mathrm{CD} 45 \mathrm{RA}+\mathrm{CD} 45 \mathrm{RO}+$ & $24.06 \pm 10.46$ & $20.28 \pm 7.79$ & 0.117 \\
\hline $\mathrm{CD} 8+$ naïve/memory ratio & $1.46 \pm 0.57$ & $1.30 \pm 0.73$ & 0.450 \\
\hline
\end{tabular}

NSCLC $=$ non-small cell lung cancer, $\mathrm{SD}=$ standard deviation.

Table 3: Associations between immune parameters and clinicopathologic parameters in 76 NSCLC patients

\begin{tabular}{|c|c|c|c|c|c|c|c|c|c|c|c|c|c|c|c|c|}
\hline \multirow{2}{*}{ Variable } & \multicolumn{2}{|c|}{ Naïve CD4+ } & \multicolumn{2}{|c|}{ Memory CD4+ } & \multicolumn{2}{|c|}{$\begin{array}{c}\text { CD4+CD45RA }+ \\
\text { CD45RO+ }\end{array}$} & \multicolumn{2}{|c|}{$\begin{array}{c}\text { CD4+ naïve/ } \\
\text { memory ratio }\end{array}$} & \multicolumn{2}{|c|}{ Naïve CD8+ } & \multicolumn{2}{|c|}{ Memory CD8+ } & \multicolumn{2}{|c|}{$\begin{array}{c}\text { CD8+CD45RA }+ \\
\text { CD45RO+ }\end{array}$} & \multicolumn{2}{|c|}{$\begin{array}{l}\text { CD8+ naïve/ } \\
\text { memory ratio }\end{array}$} \\
\hline & Mean \pm SD & $P$ & Mean \pm SD & $P$ & $\operatorname{Mean} \pm$ SD & $P$ & $\operatorname{Mean} \pm$ SD & $P$ & Mean \pm SD & $P$ & Mean \pm SD & $P$ & Mean \pm SD & $P$ & Mean \pm SD & $P$ \\
\hline \multicolumn{17}{|l|}{ Age(years) } \\
\hline$<64.5$ & $39.15 \pm 15.40$ & 0.051 & $73.47 \pm 14.97$ & 0.062 & $12.69 \pm 5.29$ & 0.377 & $0.61 \pm 0.41$ & 0.056 & $65.32 \pm 19.40$ & 0.216 & $54.43 \pm 19.64$ & 0.173 & $19.82 \pm 6.18$ & 0.646 & $1.52 \pm 1.00$ & 0.054 \\
\hline$\geq 64.5$ & $28.90 \pm 14.19$ & & $82.32 \pm 12.13$ & & $11.29 \pm 4.01$ & & $0.39 \pm 0.26$ & & $58.80 \pm 11.73$ & & $62.21 \pm 13.77$ & & $21.08 \pm 8.67$ & & $1.03 \pm 0.45$ & \\
\hline \multicolumn{17}{|l|}{ Gender } \\
\hline Female & $35.98 \pm 12.98$ & 0.417 & $76.77 \pm 11.68$ & 0.531 & $12.84 \pm 4.26$ & 0.396 & $0.50 \pm 0.26$ & 0.685 & $58.98 \pm 18.76$ & 0.597 & $59.49 \pm 20.45$ & 0.984 & $18.53 \pm 5.79$ & 0.324 & $1.22 \pm 0.78$ & 0.97 \\
\hline Male & $31.30 \pm 16.09$ & & $80.03 \pm 14.52$ & & $11.4 \pm 4.59$ & & $0.45 \pm 0.37$ & & $61.99 \pm 13.66$ & & $59.36 \pm 14.88$ & & $21.43 \pm 8.40$ & & $1.21 \pm 0.72$ & \\
\hline \multicolumn{17}{|l|}{ ECOG } \\
\hline 0 & $30.52 \pm 11.86$ & 0.547 & $80.94 \pm 10.46$ & 0.557 & $11.53 \pm 4.65$ & 0.794 & $0.40 \pm 0.22$ & 0.395 & $53.54 \pm 14.29$ & 0.020 & $67.86 \pm 13.50$ & 0.017 & $21.48 \pm 6.81$ & 0.626 & $0.85 \pm 0.34$ & 0.007 \\
\hline $1-2$ & $33.77 \pm 17.02$ & & $78.09 \pm 15.37$ & & $11.95 \pm 4.50$ & & $0.50 \pm 0.39$ & & $65.46 \pm 13.92$ & & $54.62 \pm 16.03$ & & $20.14 \pm 8.41$ & & $1.41 \pm 0.81$ & \\
\hline \multicolumn{17}{|l|}{$\begin{array}{l}\text { Smoking } \\
\text { history }\end{array}$} \\
\hline No & $41.13 \pm 13.95$ & 0.024 & $72.51 \pm 12.87$ & 0.053 & $13.69 \pm 5.08$ & 0.093 & $0.61 \pm 0.29$ & 0.084 & $66.68 \pm 19.77$ & 0.146 & $54.82 \pm 22.44$ & 0.269 & $21.55 \pm 8.55$ & 0.646 & $1.58 \pm 1.04$ & 0.042 \\
\hline Yes & $28.84 \pm 14.51$ & & $82.03 \pm 13.27$ & & $10.96 \pm 4.03$ & & $0.40 \pm 0.34$ & & $58.72 \pm 12.08$ & & $61.41 \pm 12.80$ & & $20.22 \pm 7.59$ & & $1.05 \pm 0.48$ & \\
\hline \multicolumn{17}{|l|}{ Histology } \\
\hline $\mathrm{SCC}$ & $27.51 \pm 14.20$ & 0.102 & $82.7 \pm 11.53$ & 0.246 & $10.29 \pm 4.55$ & 0.054 & $0.36 \pm 0.24$ & 0.138 & $58.25 \pm 14.52$ & 0.447 & $62.86 \pm 14.35$ & 0.394 & $21.18 \pm 8.57$ & 0.772 & $1.03 \pm 0.51$ & 0.282 \\
\hline $\mathrm{ADC}$ & $35.82 \pm 14.59$ & & $77.3 \pm 15.05$ & & $13.20 \pm 3.84$ & & $0.53 \pm 0.40$ & & $62.15 \pm 14.97$ & & $58.14 \pm 17.47$ & & $20.37 \pm 7.54$ & & $1.29 \pm 0.85$ & \\
\hline \multicolumn{17}{|c|}{ Differentiation } \\
\hline $\begin{array}{c}\text { Well/ } \\
\text { Moderate }\end{array}$ & $33.56 \pm 15.52$ & 0.243 & $78.39 \pm 13.83$ & 0.281 & $12.02 \pm 5.15$ & 0.544 & $0.49 \pm 0.37$ & 0.325 & $60.44 \pm 15.45$ & 0.643 & $60.95 \pm 15.50$ & 0.614 & $21.48 \pm 7.97$ & 0.850 & $1.12 \pm 0.56$ & 0.332 \\
\hline Poor & $26.03 \pm 14.61$ & & $84.69 \pm 13.99$ & & $10.82 \pm 3.12$ & & $0.35 \pm 0.28$ & & $63.38 \pm 14.41$ & & $57.43 \pm 19.92$ & & $20.83 \pm 8.98$ & & $1.41 \pm 1.08$ & \\
\hline \multicolumn{17}{|l|}{ T stage } \\
\hline 1 & $30.23 \pm 14.90$ & 0.626 & $82.92 \pm 11.79$ & 0.381 & $13.25 \pm 5.70$ & 0.307 & $0.40 \pm 0.29$ & 0.517 & $63.24 \pm 14.19$ & 0.662 & $54.33 \pm 17.17$ & 0.326 & $17.71 \pm 8.14$ & 0.235 & $1.35 \pm 0.74$ & 0.536 \\
\hline $2-4$ & $33.28 \pm 15.56$ & & $78.03 \pm 14.21$ & & $11.38 \pm 4.11$ & & $0.49 \pm 0.35$ & & $60.56 \pm 15.45$ & & $60.85 \pm 16.07$ & & $21.46 \pm 7.64$ & & $1.17 \pm 0.73$ & \\
\hline \multicolumn{17}{|l|}{$\mathrm{N}$ stage } \\
\hline 0 & $31.93 \pm 16.66$ & 0.882 & $80.65 \pm 16.09$ & 0.705 & $12.71 \pm 5.64$ & 0.491 & $0.48 \pm 0.48$ & 0.893 & $61.06 \pm 11.55$ & 0.982 & $57.93 \pm 15.06$ & 0.760 & $19.17 \pm 7.34$ & 0.524 & $1.17 \pm 0.52$ & 0.850 \\
\hline $1-3$ & $32.82 \pm 15.10$ & & $78.61 \pm 13.12$ & & $11.49 \pm 4.12$ & & $0.46 \pm 0.29$ & & $61.19 \pm 16.21$ & & $59.89 \pm 16.94$ & & $21.11 \pm 8.01$ & & $1.22 \pm 0.79$ & \\
\hline \multicolumn{17}{|c|}{$\begin{array}{l}\text { Clinical stage } \\
\text { (TNM) }\end{array}$} \\
\hline I-III & $31.59 \pm 13.75$ & 0.556 & $79.85 \pm 11.23$ & 0.636 & $11.52 \pm 5.02$ & 0.582 & $0.43 \pm 0.25$ & 0.310 & $61.42 \pm 15.38$ & 0.875 & $59.49 \pm 15.90$ & 0.960 & $20.99 \pm 8.45$ & 0.676 & $1.18 \pm 0.63$ & 0.757 \\
\hline IV & $34.90 \pm 18.78$ & & $77.46 \pm 18.71$ & & $12.43 \pm 3.06$ & & $0.55 \pm 0.48$ & & $60.55 \pm 14.87$ & & $59.19 \pm 17.98$ & & $19.79 \pm 6.34$ & & $1.27 \pm 0.94$ & \\
\hline
\end{tabular}

NSCLC=non-small cell lung cancer, $\mathrm{SD}=$ standard deviation, $\mathrm{ECOG}=$ Eastern Cooperative Oncology Group, $\mathrm{SCC}=$ squamous cell carcinoma, $\mathrm{ADC}=$ adenocarcinoma. 
Table 4: Progression free survival of 76 NSCLC patients stratified by immune parameters and clinicopathologic parameters

\begin{tabular}{|c|c|c|c|}
\hline Variable & Median survival (months) & HR $(95 \% \mathrm{CI})$ & P-value \\
\hline \multicolumn{4}{|l|}{ Naïve CD4+ } \\
\hline$<$ median & 10 & 1 & 0.583 \\
\hline$\geq$ median & 13 & $0.80(0.35-1.85)$ & \\
\hline \multicolumn{4}{|l|}{ Memory CD4+ } \\
\hline$<$ median & 15 & 1 & 0.138 \\
\hline$\geq$ median & 9 & $1.82(0.78-4.25)$ & \\
\hline \multicolumn{4}{|c|}{ CD4+CD45RA+CD45RO+ } \\
\hline$<12.5$ & 15 & 1 & 0.058 \\
\hline$\geq 12.5$ & 9 & $2.71(1.15-6.36)$ & \\
\hline \multicolumn{4}{|c|}{ CD4+ naïve/memory ratio } \\
\hline$<0.34$ & 9 & 1 & 0.013 \\
\hline$\geq 0.34$ & 17 & $0.47(0.20-0.92)$ & \\
\hline \multicolumn{4}{|l|}{ Naïve CD8+ } \\
\hline$<$ median & 13 & 1 & 0.808 \\
\hline$\geq$ median & 13 & $1.11(0.47-2.59)$ & \\
\hline \multicolumn{4}{|l|}{ Memory CD8+ } \\
\hline$<$ median & 13 & 1 & 0.726 \\
\hline$\geq$ median & 13 & $1.15(0.50-2.67)$ & \\
\hline \multicolumn{4}{|c|}{$\mathrm{CD} 8+\mathrm{CD} 45 \mathrm{RA}+\mathrm{CD} 45 \mathrm{RO}+$} \\
\hline$<$ median & 12 & 1 & 0.806 \\
\hline$\geq$ median & 15 & $0.90(0.39-2.11)$ & \\
\hline \multicolumn{4}{|c|}{ CD8+ naïve/memory ratio } \\
\hline$<$ median & 13 & 1 & 0.567 \\
\hline$\geq$ median & 13 & $0.79(0.34-1.84)$ & \\
\hline \multicolumn{4}{|l|}{ Gender } \\
\hline Female & 15 & 1 & 0.156 \\
\hline Male & 12 & $1.85(0.68-6.35)$ & \\
\hline \multicolumn{4}{|l|}{ Age(years) } \\
\hline$<64.5$ & 13 & 1 & 0.682 \\
\hline$\geq 64.5$ & 15 & $0.86(0.29-1.58)$ & \\
\hline \multicolumn{4}{|l|}{ Smoking status } \\
\hline No & 15 & 1 & 0.583 \\
\hline Yes & 13 & $1.25(0.68-3.15)$ & \\
\hline \multicolumn{4}{|l|}{ Differentiation } \\
\hline Well and moderate & 15 & 1 & 0.006 \\
\hline Poor & 9 & $3.95(2.35-12.37)$ & \\
\hline \multicolumn{4}{|l|}{ ECOG } \\
\hline 0 & 17 & 1 & 0.018 \\
\hline $1-2$ & 12 & $3.46(1.28-8.24)$ & \\
\hline \multicolumn{4}{|l|}{ T stage } \\
\hline 1 & 15 & 1 & 0.482 \\
\hline $2-4$ & 13 & $1.82(0.79-6.25)$ & \\
\hline \multicolumn{4}{|l|}{$\mathrm{N}$ stage } \\
\hline 0 & 17 & 1 & 0.026 \\
\hline $1-3$ & 13 & $3.28(1.13-9.67)$ & \\
\hline \multicolumn{4}{|l|}{ Clinical stage (TNM) } \\
\hline I-III & 15 & 1 & 0.004 \\
\hline IV & 9 & $3.68(1.45-8.47)$ & \\
\hline
\end{tabular}

$\mathrm{NSCLC}=$ non-small cell lung cancer, $\mathrm{HR}=$ hazard ratio, $\mathrm{CI}=$ confidence interval, $\mathrm{ECOG}=$ Eastern Cooperative Oncology Group performance status. 
Table 5: Multivariate cox proportional hazard models for progression free survival of 76 NSCLC patients

\begin{tabular}{lccc}
\hline Variable & HR & $\mathbf{9 5 \% C I}$ & P-value \\
\hline N stage & & & 0.002 \\
$\quad$ 1-3 vs 0 & 10.79 & $2.43-47.85$ & 0.016 \\
$\begin{array}{l}\text { Clinical stage } \\
\quad \text { IV vs I-III }\end{array}$ & & \\
ECOG & 4.61 & $1.34-15.91$ & 0.135 \\
$\quad$ 1-2 vs 0 & 2.77 & $0.82-9.35$ & \\
$\begin{array}{l}\text { Differentiation } \\
\quad \text { Poor vs Well and moderate }\end{array}$ & 3.34 & $0.84-13.33$ & 0.087 \\
CD4+ naïve/memory ratio & & & \\
$\quad \geq 0.34$ vs $<0.34$ & 0.35 & $0.19-0.75$ & 0.012 \\
\hline
\end{tabular}

NSCLC=non-small cell lung cancer, ECOG=Eastern Cooperative Oncology Group.

tumor-infiltrating lymphocytes in tumor tissues [19-21]. Several possibilities may explain above phenomena. First, in tumor tissues, memory cells elicited robust immune response to attack tumor cells [13]. However, memory CD4+ cells in peripheral blood showed lower proliferation than primary responding cells when they reencountered antigen and failed to elicit robust immune response [22-24]. In contrast, naïve $\mathrm{T}$ cells were more capable of proliferation and anti-tumor efficiency [25]. Besides, high levels of $\mathrm{CD} 45 \mathrm{RO}+$ tumor-infiltrating lymphocytes were associated with low tumor stage and invasion [26]. Correlations between elevated CD4+CD45RA $+\mathrm{CD} 45 \mathrm{RO}+$ cells and poor survival were observed with a trend in univariate analysis. $\mathrm{CD} 4+\mathrm{CD} 45 \mathrm{RA}+\mathrm{CD} 45 \mathrm{RO}+$ cells transitioned from naïve to memory cells after stimulation by tumor antigen;

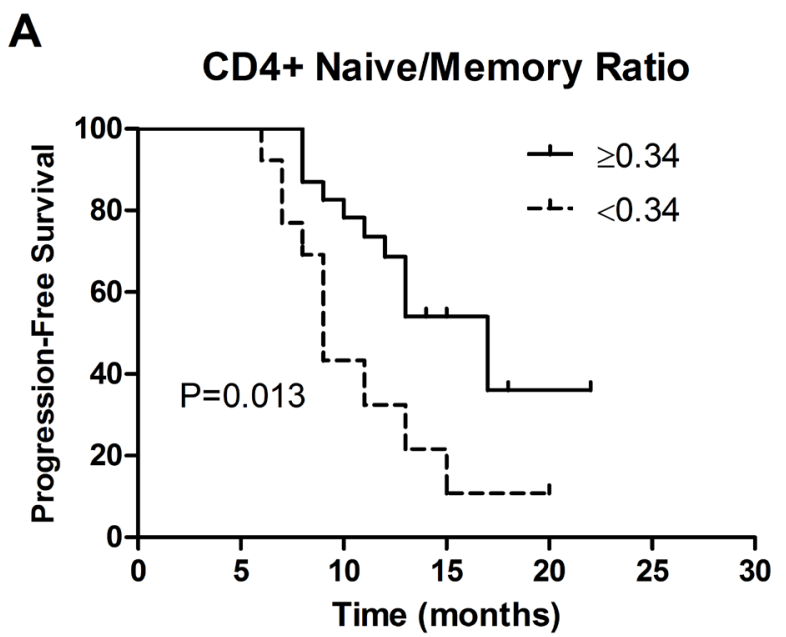

increased proportions of these cells may suggest higher tumor malignancy and invasion.

Furthermore, we found no predictive roles of naïve $\mathrm{CD} 8+\mathrm{T}$ cells, memory $\mathrm{CD} 8+\mathrm{T}$ cells, $\mathrm{CD} 8+$ naïve/memory ratio, and $\mathrm{CD} 8+\mathrm{CD} 45 \mathrm{RA}+\mathrm{CD} 45 \mathrm{RO}+$ $\mathrm{T}$ cells, which agrees with previous studies [17, 18]. Decreased percentages of naïve CD4 $+\mathrm{T}$ cells, naïve $\mathrm{CD} 8+\mathrm{T}$ cells, $\mathrm{CD} 4+$ naïve/memory ratios, and $\mathrm{CD} 4+\mathrm{CD} 45 \mathrm{RA}+\mathrm{CD} 45 \mathrm{RO}+\mathrm{T}$ cells and increased memory $\mathrm{CD} 4+\mathrm{T}$ cells were observed in NSCLC patients, suggesting that the immune system was activated against cancer. A previous study has not found these results with statistical significance, which may have been accounted for by the small number of patients and early tumor grade [17]. In nonsmokers and young patients, increased percentages of naïve $\mathrm{CD} 4+\mathrm{T}$ cells

B

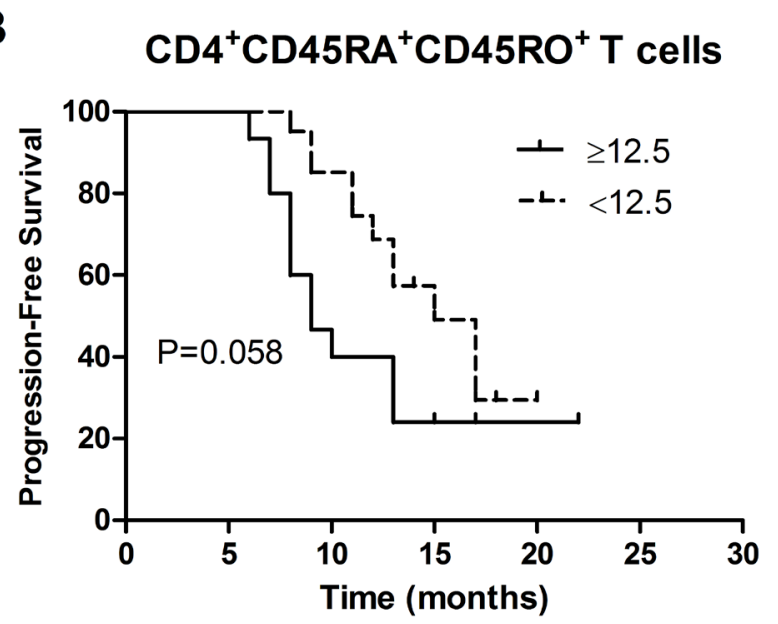

Figure 2: Progression-free survival of 76 NSCLC patients stratified by (A) CD4+ naïve/memory ratio; (B) CD4+CD45RA+CD45RO+ $\mathrm{T}$ cells. 
and decreased memory CD4+ T cells were observed, which were consistent with tumor characteristics of these patients.

The limitations of this study include small sample size, different clinical stages, different treatment strategies and a short follow-up time that results in the lack of detailed information on overall survival. Despite these weaknesses, we documented that CD4+ naïve/ memory ratio independently predicted tumor progression. Moreover, decreased proportions of naïve CD4+ T cells, naïve $\mathrm{CD} 8+\mathrm{T}$ cells, $\mathrm{CD} 4+$ naïve/memory ratio and CD4+CD45RA+CD45RO+ T cells, and increased memory CD4+ T cells were observed in NSCLC patients compared to healthy controls.

In summary, peripheral CD4+ naïve/memory ratio may provide an independent predictor of tumor progression in NSCLC patients. This information may provide opportunities to select NSCLC patients who would have poor survival, and optimize treatment strategies for them.

\section{MATERIALS AND METHODS}

\section{Patients selection}

We prospectively included 76 NSCLC patients with histological confirmation, who were treated in Linyi People's Hospital. The inclusion criteria for patients were as follows: They had not received anti-tumor therapies or other therapies that influence patients' immune status before enrollment and were over 18 years old; they had no infections, other cancers, immune system related diseases or transplant history. Twenty-eight age- and sex-matched healthy volunteers were included. Clinicopathologic characteristics of the enrolled NSCLC patients were collected from the electronic medical record system in our hospital. Clinical stage was evaluated by American Joint Committee on Cancer-7 criteria. Informed consent was obtained from all patients and volunteers. The present study was approved by the Ethical Committee of Linyi People's Hospital. All methods in the present study were approved by the committee and performed in accordance with relevant guidelines and regulations.

\section{Treatment protocol and follow-up}

Early stage patients received radical surgery and lymph node dissection; advanced patients received radiotherapy combined with chemotherapy/targeted therapy concurrently or consecutively. First-line chemotherapy was with platinum-based agents; gefitinib, erlotinib, and crizotinib were used for targeted therapy. Radiotherapy was delivered with 60-66 Gy/30-33 fractions by linear accelerator. Patients were followed up regularly per 3 months after treatment. Follow-up data collection ended on April 16, 2017 or the time of death of the patient.
The median follow-up was 13 months (range 6-22.5 months). The primary endpoint was PFS, which was defined as the interval between the date of initial therapy and the date of the first RECIST- (Response Evaluation Criteria in Solid Tumors) 1.1 [27] defined progression, death, or loss to follow-up.

\section{Detection of immune cells}

Fresh peripheral blood samples obtained from patients prior to cancer treatment and from volunteers were stored in EDTA anticoagulation tubes before testing. Four specific monoclonal antibodies (mAbs) against CD4 (FITC and APC), CD8 (FITC and APC), CD45RA (FITC), CD45RO (PE) were used to differentiate immune cells. Initially, $200 \mu$ l blood was mixed with the above mAbs and incubated in the dark at room temperature. By using FACS lysing solution (BD Biosciences, San Jose, CA, USA), red blood cells in the mix were lysed and then washed twice with phosphate buffered saline (PBS). The residual white blood cells were analyzed by flow cytometry and the percentages of these immune cells were calculated by FlowJo Version 10.0 data analysis software (FlowJo, Ashland, OR, USA).

Immune cells were identified as follow: naïve CD4+ T cells (CD4+CD45RA +$)$, memory CD4+ T cells (CD4+CD45RO+), naïve CD8+ T cells (CD8+CD45RA+), memory CD8+ T cells (CD8+CD45RO+), CD4+CD4 $5 \mathrm{RA}+\mathrm{CD} 45 \mathrm{RO}+\mathrm{T}$ cells $(\mathrm{CD} 4+\mathrm{CD} 45 \mathrm{RA}+\mathrm{CD} 45 \mathrm{RO}+)$ and $\mathrm{CD} 8+\mathrm{CD} 45 \mathrm{RA}+\mathrm{CD} 45 \mathrm{RO}+\mathrm{T}$ cells $(\mathrm{CD} 8+\mathrm{CD} 45 \mathrm{RA}+$ $\mathrm{CD} 45 \mathrm{RO}+$ ). The percentages of immune cells were expressed as proportions of CD4+/CD8+ T cells.

\section{Statistical analysis}

Percentages of immune cells were expressed with mean \pm standard deviation (SD). Differences of immune cells between NSCLC patients and controls, and relationships between patient characteristics and immune cells were determined by the Student's $t$-test. The Kaplan-Meier analysis was employed to estimate PFS and compare PFS between groups by log-rank test. The Cox proportional hazards model was performed to determine hazard ratios (HRs) and 95\% confidence intervals (CIs). Variables of $P<0.05$ in univariate analysis were enrolled in multiple analysis. Data analyses were performed using the Statistical Package for Social Sciences, Version 22.0 (IBM Corporation, Armonk, NY, USA) and GraphPad Version 5.0 (GraphPad Software Inc., San Diego, CA, USA); $P<0.05$ was defined as statistically significant.

\section{CONFLICTS OF INTEREST}

The authors report no conflicts of interest in this work. 


\section{REFERENCES}

1. Chen W, Zheng R, Baade PD, Zhang S, Zeng H, Bray F, Jemal A, Yu XQ, He J. Cancer statistics in China, 2015. CA Cancer J Clin. 2016; 66:115-32. doi: 10.3322/caac.21338.

2. Arriagada R, Auperin A, Burdett S, Higgins JP, Johnson DH, Le Chevalier T, Le Pechoux C, Parmar MK, Pignon JP, Souhami RL, Stephens RJ, Stewart LA, Tierney JF, et al. Adjuvant chemotherapy, with or without postoperative radiotherapy, in operable non-small-cell lung cancer: two meta-analyses of individual patient data. Lancet. 2010; 375:1267-77. doi: 10.1016/s0140-6736(10)60059-1.

3. Scagliotti GV, Pastorino U, Vansteenkiste JF, Spaggiari L, Facciolo F, Orlowski TM, Maiorino L, Hetzel M, Leschinger M, Visseren-Grul C, Torri V. Randomized phase III study of surgery alone or surgery plus preoperative cisplatin and gemcitabine in stages IB to IIIA non-small-cell lung cancer. J Clin Oncol. 2012; 30:172-8. doi: 10.1200/ jco.2010.33.7089.

4. Zeng J, Baik C, Bhatia S, Mayr N, Rengan R. Combination of stereotactic ablative body radiation with targeted therapies. Lancet Oncol. 2014; 15:e426-34. doi: 10.1016/ s1470-204570026-9.

5. Jaffray DA. Image-guided radiotherapy: from current concept to future perspectives. Nat Rev Clin Oncol. 2012; 9:688-99. doi: 10.1038/nrclinonc.2012.194.

6. Vesely MD, Kershaw MH, Schreiber RD, Smyth MJ. Natural innate and adaptive immunity to cancer. Annu Rev Immunol. 2011; 29:235-71. doi: 10.1146/ annurev-immunol-031210-101324.

7. Dunn GP, Old LJ, Schreiber RD. The three Es of cancer immunoediting. Annu Rev Immunol. 2004; 22:329-60. doi: 10.1146/annurev.immunol.22.012703.104803.

8. Tokito T, Azuma K, Kawahara A, Ishii H, Yamada K, Matsuo N, Kinoshita T, Mizukami N, Ono H, Kage M, Hoshino T. Predictive relevance of PD-L1 expression combined with CD8+ TIL density in stage III nonsmall cell lung cancer patients receiving concurrent chemoradiotherapy. Eur J Cancer. 2016; 55:7-14. doi: 10.1016/j.ejca.2015.11.020.

9. Kinoshita T, Muramatsu R, Fujita T, Nagumo H, Sakurai T, Noji S, Takahata E, Yaguchi T, Tsukamoto N, Kudo-Saito C, Hayashi Y, Kamiyama I, Ohtsuka T, et al. Prognostic value of tumor-infiltrating lymphocytes differs depending on histological type and smoking habit in completely resected non-small cell lung cancer. Ann Oncol. 2016; 27:2117-23. doi: 10.1093/annonc/mdw319.

10. Saavedra D, Garcia B, Lorenzo-Luaces P, Gonzalez A, Popa X, Fuentes KP, Mazorra Z, Crombet T, Neninger E, Lage A. Biomarkers related to immunosenescence: relationships with therapy and survival in lung cancer patients. Cancer Immunol Immunother. 2016; 65:37-45. doi: 10.1007/ s00262-015-1773-6.
11. Kotsakis A, Koinis F, Katsarou A, Gioulbasani M, Aggouraki D, Kentepozidis N, Georgoulias V, Vetsika EK. Prognostic value of circulating regulatory $\mathrm{T}$ cell subsets in untreated non-small cell lung cancer patients. Sci Rep. 2016; 6:39247. doi: 10.1038/srep39247.

12. Ke X, Zhang S, Xu J, Liu G, Zhang L, Xie E, Gao L, Li D, Sun R, Wang F, Pan S. Non-small-cell lung cancerinduced immunosuppression by increased human regulatory $\mathrm{T}$ cells via Foxp3 promoter demethylation. Cancer Immunol Immunother. 2016; 65:587-99. doi: 10.1007/ s00262-016-1825-6.

13. Farber DL, Yudanin NA, Restifo NP. Human memory T cells: generation, compartmentalization and homeostasis. Nat Rev Immunol. 2014; 14:24-35. doi: 10.1038/nri3567.

14. Michie CA, McLean A, Alcock C, Beverley PC. Lifespan of human lymphocyte subsets defined by CD45 isoforms. Nature. 1992; 360:264-5. doi: 10.1038/360264a0.

15. MacLeod MK, Kappler JW, Marrack P. Memory CD4 T cells: generation, reactivation and re-assignment. Immunology. 2010; 130:10-5. doi: 10.1111/j.1365-2567.2010.03260.x.

16. LaSalle JM, Hafler DA. The coexpression of CD45RA and CD45RO isoforms on $\mathrm{T}$ cells during the $\mathrm{S} / \mathrm{G} 2 / \mathrm{M}$ stages of cell cycle. Cell Immunol. 1991; 138:197-206.

17. Hara M, Matsuzaki Y, Shimizu T, Tomita M, Ayabe T, Enomoto Y, Onitsuka T. Preoperative peripheral naive/ memory ratio and prognosis of nonsmall-cell lung cancer patients. Ann Thorac Cardiovasc Surg. 2007; 13:384-90.

18. Prado-Garcia H, Aguilar-Cazares D, Flores-Vergara H, Mandoki JJ, Lopez-Gonzalez JS. Effector, memory and naive CD8+ T cells in peripheral blood and pleural effusion from lung adenocarcinoma patients. Lung Cancer. 2005; 47:361-71. doi: 10.1016/j.lungcan.2004.07.046.

19. Kilvaer TK, Paulsen EE, Khanehkenari MR, Al-Saad S, Johansen RM, Al-Shibli K, Bremnes RM, Busund LT, Donnem T. The presence of intraepithelial CD45RO+ cells in resected lymph nodes with metastases from NSCLC patients is an independent predictor of disease-specific survival. Br J Cancer. 2016; 114:1145-51. doi: 10.1038/ bjc.2016.92.

20. Paulsen EE, Kilvaer T, Khanehkenari MR, Maurseth RJ, Al-Saad S, Hald SM, Al-Shibli K, Andersen S, Richardsen E, Busund LT, Bremnes R, Donnem T. CD45RO(+) memory $\mathrm{T}$ lymphocytes--a candidate marker for TNM-immunoscore in squamous non-small cell lung cancer. Neoplasia. 2015; 17:839-48. doi: 10.1016/j.neo.2015.11.004.

21. Wang W, Hodkinson P, McLaren F, Mackean MJ, Williams L, Howie SE, Wallace WA, Sethi T. Histologic assessment of tumor-associated CD45(+) cell numbers is an independent predictor of prognosis in small cell lung cancer. Chest. 2013; 143:146-51. doi: 10.1378/chest.12-0681.

22. MacLeod MK, McKee A, Crawford F, White J, Kappler J, Marrack P. CD4 memory $\mathrm{T}$ cells divide poorly in 
response to antigen because of their cytokine profile. Proc Natl Acad Sci U S A. 2008; 105:14521-6. doi: 10.1073/ pnas.0807449105.

23. David A, Crawford F, Garside P, Kappler JW, Marrack P, MacLeod M. Tolerance induction in memory CD4 T cells requires two rounds of antigen-specific activation. Proc Natl Acad Sci U S A. 2014; 111:7735-40. doi: 10.1073/ pnas. 1406218111.

24. Klebanoff CA, Scott CD, Leonardi AJ, Yamamoto TN, Cruz AC, Ouyang C, Ramaswamy M, Roychoudhuri R, Ji Y, Eil RL, Sukumar M, Crompton JG, Palmer DC, et al. Memory T cell-driven differentiation of naive cells impairs adoptive immunotherapy. J Clin Invest. 2016; 126:318-34. doi: $10.1172 /$ jci81217.

25. Hinrichs CS, Borman ZA, Gattinoni L, Yu Z, Burns WR, Huang J, Klebanoff CA, Johnson LA, Kerkar SP, Yang S, Muranski P, Palmer DC, Scott CD, et al. Human effector CD8 $+\mathrm{T}$ cells derived from naive rather than memory subsets possess superior traits for adoptive immunotherapy. Blood. 2011; 117:808-14. doi: 10.1182/ blood-2010-05-286286.

26. Pages F, Berger A, Camus M, Sanchez-Cabo F, Costes A, Molidor R, Mlecnik B, Kirilovsky A, Nilsson M, Damotte D, Meatchi T, Bruneval P, Cugnenc PH, et al. Effector memory T cells, early metastasis, and survival in colorectal cancer. N Engl J Med. 2005; 353:2654-66. doi: 10.1056/ NEJMoa051424.

27. Eisenhauer EA, Therasse P, Bogaerts J, Schwartz LH, Sargent D, Ford R, Dancey J, Arbuck S, Gwyther S, Mooney M, Rubinstein L, Shankar L, Dodd L, et al. New response evaluation criteria in solid tumours: revised RECIST guideline (version 1.1). Eur J Cancer. 2009; 45:228-47. doi: 10.1016/j.ejca.2008.10.026. 